\title{
The Effectiveness of a Systematic Algorithm for the Management of Vascular Injuries during the Laparoscopic Surgery
}

\author{
Carlo C. Passerottia, José A. Cruz ${ }^{\mathrm{a}, \mathrm{b}} \quad$ Sabrina T. Reis ${ }^{\mathrm{a}} \quad$ Marcelo T. Okano $^{\mathrm{a}}$ \\ Ricardo J. Duarte ${ }^{\mathrm{a}}$ Miguel Srougi $^{\mathrm{a}}$ Hiep T. Nguyen ${ }^{\mathrm{c}}$

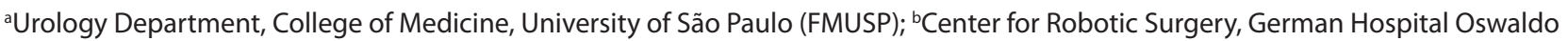 \\ Cruz, São Paulo, Brazil; ' Urology Department, Cardon Children Medical Center, Mesa, Ariz., USA
}

\section{Key Words}

Laparoscopy $\cdot$ Complication • Blood loss • Training

\begin{abstract}
Objectives: Currently, there is no standardized training protocol to teach surgeons how to deal with vascular injuries during laparoscopic procedures. The purpose of this study is to develop and evaluate the effectiveness of a standardized algorithm for managing vascular injury during laparoscopic nephrectomies. Materials and Methods: The performance of 6 surgeons was assessed during 10 laparoscopic nephrectomies in a porcine model. During the first and tenth operations, an injury was made in the renal vein without warning the surgeon. After the first procedure, the surgeons were instructed on how to proceed in dealing with the vascular injury, according to an algorithm developed by the designers of this study. The performance of each surgeon before and after learning the algorithm was assessed. Results: After learning the algorithm there was a decreased blood loss from $327 \pm 403.11 \mathrm{ml}$ to $37 \pm 18.92 \mathrm{ml}(p=0.031)$ and decreased operative time from $43 \pm 14.53 \mathrm{~min}$ to $27 \pm 8.27$ $\min (p=0.015)$. There was also improvement in the time to start lesion repair from $147 \pm 117.65 \mathrm{sec}$ to $51 \pm 39.09 \mathrm{sec}$ $(p=0.025)$. There was a trend toward improvement in the
\end{abstract}

\section{KARGER}

Fax +4161306 1234

E-Mail karger@karger.ch

www.karger.com
(C) 2015 S. Karger AG, Basel

1015-9770/15/0093-0138\$38.00/0

Accessible online at:

www.karger.com/cur reaction time to the injury $(22 \pm 21.55$ sec vs. $14 \pm 6.39, p=$ $0.188)$, the time required to control the bleeding ( $50 \pm 94.2$ sec vs. $14 \pm 6.95 \mathrm{sec}, \mathrm{p}=0.141)$, and the total time required to completely repair of the vascular injury (178 $\pm 170.4 \mathrm{sec}$ vs. $119 \pm 183.87 \mathrm{sec}, \mathrm{p}=0.302$ ). Conclusion: $\mathrm{A}$ standardized algorithm may help to reduce the potential risks associated with laparoscopic surgery. Further studies will help to refine and determine the benefits of standardized protocols such as that developed in this study for the management of life-threatening laparoscopic complications.

Copyright $\odot 2015$ S. Karger AG, Basel

\section{Introduction}

Minimally invasive surgery (MIS) has provided surgeons with the opportunity to perform complex procedures with less morbidity and good outcomes. However, it is not without its associated potential complications. The types of complications range from more minor ones such as difficulty accessing the abdominal cavity (incidence rates $0.2-3.1 \%$ [1-3]) to those with significant morbidity such as vascular and organ injuries (incidence rates from $0.5 \%$ to as high as $20 \%$ depending on the type 
Table 1. Algorithm for the management of vascular injuries during minimally invasive surgeries

\begin{tabular}{ll}
\hline Algorithm & Description \\
\hline A-air pressure & $\begin{array}{l}\text { The intra-abdominal pressure created by the gas insufflation must be increased to decrease the venous } \\
\text { return and decrease bleeding. }\end{array}$ \\
B-blood aspiration & $\begin{array}{l}\text { Any blood or other secretions released into the cavity should be aspirated to allow visualization of the } \\
\text { injured site and to prevent infections. Aspiration should be continued until the lesion is completely } \\
\text { repaired. Addition ports may be needed to accommodate the addition of the suction-irrigation device. }\end{array}$ \\
C-clamping & $\begin{array}{l}\text { In the case of bleeding or secretion leakage, the site must be clamped to stop the bleeding/extravasation. } \\
\text { D-display lesion }\end{array}$ \\
$\begin{array}{l}\text { After the bleeding/leakage has stopped, the next step is to visualize the lesion site to make sure that } \\
\text { there is no more leakage of any substance and to actively search for another possible injury. }\end{array}$ \\
After ensuring that there is no other injury and no leakage, the next step is to repair all the existing \\
famage by suture, clips or any means necessary. \\
The surgeon must ascertain whether it is possible to finish the procedure by laparoscopy or if it would \\
be wiser to convert the procedure to an open surgery.
\end{tabular}

of MIS [4-6]). While these complications rates may appear low, the number of patients affected is great, given the number of MIS procedures performed each year. For example, the complication rate for laparoscopic cholecystectomies, one of the first routinely performed MIS procedures, is approximately $1.5 \%$, with a mortality rate of approximately $0.1 \%$. In the United States alone, 500,000 cholecystectomies are performed annually, translating into 7,500 cases with complications and 500 deaths annually for a surgical procedure that is considered simple and easy to perform [7]. Consequently, it is essential that any surgeon who performs MIS should be well trained to deal with the potential complications, especially serious ones such as vascular injuries [8].

Sponsored by the Society of American Gastrointestinal and endoscopic Surgeons and the American College of Surgeons, the Fundamentals of Laparoscopic Surgery (FLS) program was developed from the need to safely introduce laparoscopic techniques to clinical practice and to demonstrate basic competence in MIS. Implementation of the FLS program appears to be effective in improving laparoscopic surgical performance $[9,10]$. However, management of MIS complications such as vascular injury is lacking in the FLS program. Unfortunately, most surgeons' first exposure to the management of MIS complications is in the operating room during an actual case.

In the 1970s, the American College of Surgeons Committee on Trauma proposed a standardized method, the Advanced Trauma Life Support algorithm, for the initial management of a trauma patient using a simple mnemonic sequence of letters [11]. Inspired by the Advanced
Trauma Life Support algorithm, we proposed the creation of a systematic approach to the management of laparoscopic vascular injury and evaluate its effectiveness.

\section{Materials and Methods}

This was a prospective controlled study, with each surgeon serving as its own control. This study was approved by the ethics committee of our institution. The participants were informed and consented to be evaluated but were not made aware of the events to occur during the procedure or the exact performance parameters being assessed.

\section{Algorithm}

The algorithm begins with ensuring the availability of equipment (such as suction-irrigation devices, graspers/forceps, vascular clamps and sutures) and having adequate lighting prior to beginning the procedure. Next, a series of steps with the mnemonic, ABCDEF, is employed in the advent of a vascular complication (table 1).

\section{Surgical Assessment}

The surgical performances of 6 newly trained surgeons (finishing a clinical fellowship in Urologic Oncology in the University of São Paulo) who had mild to moderate experience in laparoscopy were evaluated; each surgeon was asked to perform 10 radical laparoscopic nephrectomies in a porcine model. All procedures were recorded. The animals were anesthetized with Telazol (4.4-6.6 $\mathrm{mg} / \mathrm{kg}$ intramuscularly), xylazine $(1.1-2.2 \mathrm{mg} / \mathrm{kg}$ intramuscularly) and atropine $(0.04 \mathrm{mg} / \mathrm{kg}$ intramuscularly). Anesthesia was maintained with isoflurane (1-4\%) mixed with 1-2 liters of oxygen through an endotracheal tube. After the procedures, the pigs were sacrificed.

An intentional standardized injury was made in the renal vein (complete section with a scissor) without the surgeon's foreknowledge (the surgeon was asked to switch his instruments for 


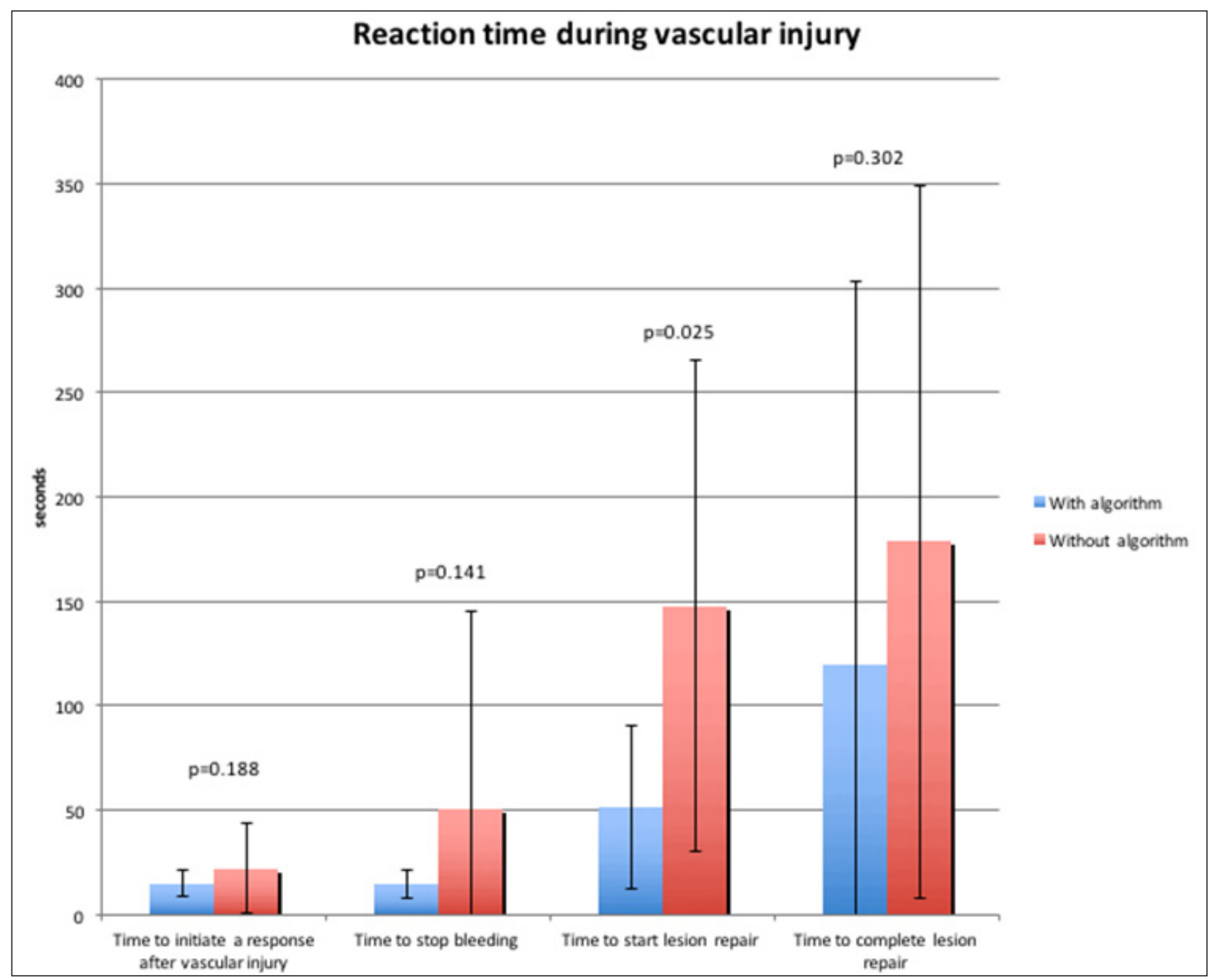

Fig. 1. Reaction time during vascular injury.

new ones, while the assisting surgeon injured the renal vein) during the 1 st and 10th procedure. The participants were taught the above algorithm after the 1st procedure.

Surgical parameters evaluated included the following:

1) Reaction time (defined as elapsed time from injury until the moment the surgeon initiated a response);

2) Bleeding time (defined as time between the initial reaction and control of the bleeding vessel);

3) Repair start time (defined as the time between control of the bleeding vessel and placement of the first clip on the renal vein);

4) Repair completion time (defined as the time between placing the first and last clips to repair the damaged renal vein);

5) Total operative time;

6) Aspirated blood loss.
Comparison of these parameters was then made between the 1 st and 10th procedures through t-paired test, since that data had normal distribution. A p-value of 0.05 was considered to be statistically significant. The software used was StatPlus ${ }^{\circledR}$ v. 2009 for Mac.

\section{Results}

With the introduction of the algorithm, there was improvement in all parameters assessed (fig. 1-3): a reduction in aspirated blood loss from $327 \pm 403.11 \mathrm{ml}$ to 37 $\pm 18.92 \mathrm{ml}(\mathrm{p}=0.031)$, a decrease in the total operative 


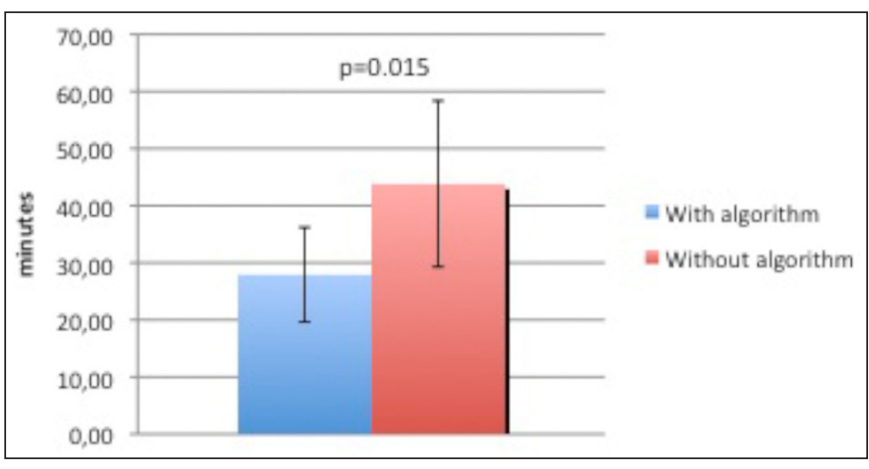

Fig. 2. Total operative time.

time from $43 \pm 14.53 \min$ to $27 \pm 8.27 \min (\mathrm{p}=0.015)$ and an improvement in the repair start time from $147 \pm$ $117.65 \mathrm{sec}$ to $51 \pm 39.09(\mathrm{p}=0.025)$. There was a trend toward improvement in the reaction time $(22 \pm 21.55 \mathrm{sec}$ vs. $14 \pm 6.39 \mathrm{sec}, \mathrm{p}=0.188)$, the bleeding time $(50 \pm$ $94.2 \mathrm{sec}$ vs. $14 \pm 6.95 \mathrm{sec}, \mathrm{p}=0.141$ ), and the repair completion time $(178 \pm 170.4 \mathrm{sec}$ vs. $119 \pm 183.87 \mathrm{sec}$, $\mathrm{p}=0.302$ ).

\section{Discussion}

Management of vascular injuries during MIS is often difficult due to the limited visibility and morbidity produced by these procedures [12]. Training is essential in the acquisition and mastery of any surgical skill [13], particularly in laparoscopic surgery and in managing trauma, complications and other emergencies. Adopting a standardized protocol to systematize the surgeon's response to such situations allows for more rapid recall of key principles and appropriate responses while under intense pressure or stress [14]. In this study, we observed that by learning and utilizing a simple mnemonic, inexperienced surgeons were able to significantly limit blood loss following inadvertent vascular injury during laparoscopic surgery. The use of the algorithm appears to facilitate the surgeons to focus more rapidly and effectively on control and repair of the injury. Interestingly, we also observed that the standard deviations associated with most surgical parameters assessed were much lower in those procedures in which the surgeon was taught the algorithm. This finding may suggest that the algorithm not only optimizes the responses of surgeons but also makes them more uniform. To our knowledge, no prior

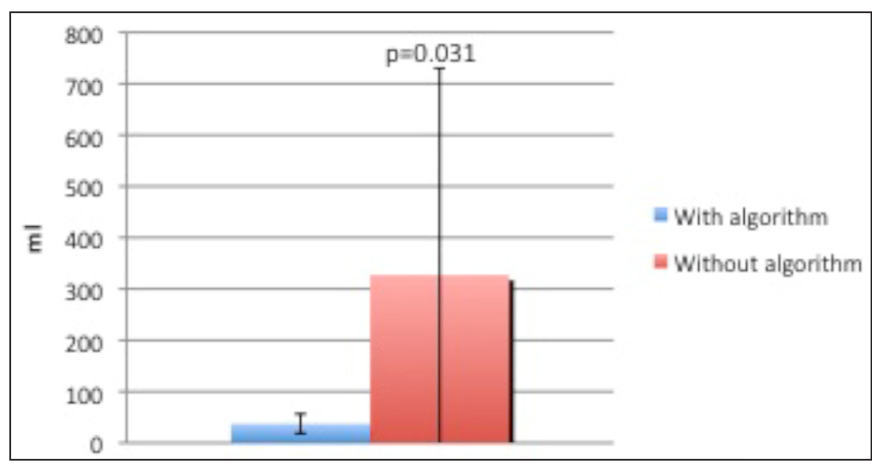

Fig. 3. Aspirated blood loss.

algorithm has been proposed in the management of vascular injuries during laparoscopic procedures.

This study has several limitations. Because all of the surgeons who participated in the study had mild to moderate operating experience, we assumed that they were all at the same level of their learning curves in performing laparoscopic procedures and that the design of the study eliminated any influence of learning prior to these 10 procedures (so that the only modifying factor for the performance of the procedures should have been the knowledge and use of the algorithm). A second limitation is the small number of participants, which decreased the statistical power of the study. However, we were still able to observe statistically significant differences between the groups despite the small numbers. Thirdly, we primarily used quantitative parameters to compare the groups, assessing the time to perform each step of injury repair. Qualitative assessment such as the quality of the repair could not be easily evaluated due to the lack of an objective means to perform such an assessment. Finally, since all our injuries were standardized, this fact by itself could have improved the response to the injury.

Our results suggest that there is benefit in the use of this algorithm, but more complex studies need to be conducted to confirm these conclusions, refine the algorithm, and to test this algorithm in different emergency situations.

\section{Conclusion}

Our findings suggest that the implementation of a standardized, easy-to-remember algorithm for the management of vascular injuries may help to reduce the po- 
tential morbidity and mortality associated with laparoscopic surgery. Further studies will help to determine the potential benefits of standardized training protocols such as that developed in this study for the management of life-threatening laparoscopic complications.

\section{Acknowledgements}

This study was funded by FAPESP (Fundacao de Amparo a Pesquisa do Estado de Sao Paulo) and by CNPQ (National Counsel of Technological and Scientific Development).

\section{References}

1 Catarci M, Carlini M, Gentileschi P, Santoro E: Major and minor injuries during the creation of pneumoperitoneum. A multicenter study on 12,919 cases. Surg Endosc 2001;15: 566-569.

2 Gutt CN, Muller-Stich BP, Reiter MA: Success and complication parameters for laparoscopic surgery: a benchmark for natural orifice transluminal endoscopic surgery. Endoscopy 2009;41:36-41.

3 Hashizume M, Sugimachi K: Needle and trocar injury during laparoscopic surgery in Japan. Surg Endosc 1997;11:1198-1201.

4 Kenney PA, Tuerk IA: Complications of laparoscopic retroperitoneal lymph node dissection in testicular cancer. World J Urol 2008; 26:561-569.

5 Laurence JM, Lam VW, Langcake ME, Hollands MJ, Crawford MD, Pleass HC: Laparoscopic hepatectomy, a systematic review. ANZ J Surg 2007;77:948-953.

6 Rassweiler JJ, Teber D, Frede T: Complications of laparoscopic pyeloplasty. World $\mathrm{J}$ Urol 2008;26:539-547.
7 Osborne DA, Alexander G, Boe B, Zervos EE: Laparoscopic cholecystectomy: past, present, and future. Surg Technol Int 2006; 15:81-85.

8 Cruz JA, Passerotti CC, Frati RM, Reis ST, Okano MT, Gouveia EM, Biolo KD, Duarte RJ, Nguyen H, Srougi M: Surgical performance during laparoscopic radical nephrectomy is improved with training in a porcine model. J Endourol 2012;26:278-282.

9 Mashaud LB, Castellvi AO, Hollett LA, Hogg DC, Tesfay ST, Scott DJ: Two-year skill retention and certification exam performance after fundamentals of laparoscopic skills training and proficiency maintenance. Surgery 2010;148:194-201.

10 Sroka G, Feldman LS, Vassiliou MC, Kaneva PA, Fayez R, Fried GM: Fundamentals of laparoscopic surgery simulator training to proficiency improves laparoscopic performance in the operating room-a randomized controlled trial. Am J Surg 2010;199:115-120.
11 van Olden GD, Meeuwis JD, Bolhuis HW, Boxma H, Goris RJ: Clinical impact of advanced trauma life support. Am J Emerg Med 2004;22:522-525.

12 Richstone L, Seideman C, Baldinger L, Permpongkosol S, Jarrett TW, Su LM, Pavlovich C, Kavoussi LR: Conversion during laparoscopic surgery: frequency, indications and risk factors. J Urol 2008;180:855-859.

13 Sugden C, Aggarwal R: Assessment and feedback in the skills laboratory and operating room. Surg Clin North Am 2010;90:519533.

14 Williams MJ, Lockey AS, Culshaw MC: Improved trauma management with advanced trauma life support (ATLS) training. J Accid Emerg Med 1997;14:81-83. 